Pacific Journal of Mathematics

REMARKS ON THE DEFECT SUM FOR A FUNCTION
MEROMORPHIC ON AN OPEN RIEMANN SURFACE 


\title{
REMARKS ON THE DEFECT SUM FOR A FUNCTION MEROMORPHIC ON AN OPEN RIEMANN SURFACE
}

\author{
PHILLIP EMIG
}

\begin{abstract}
L. Sario has extended R. Nevanlinna's concept of defect to functions defined on $W_{p}$ Riemann surfaces. He has shown that for a large class of functions the defect sum $\sum \delta(a)$ is bounded above by $2+\eta$, where $\eta$ is a number depending on the topological complexity of the surface and the rate of growth of the function under study.

By studying the relation between the rate of growth of a meromorphic function $w$ and its $P$-derivative $w_{p}=w_{z} /(d p / d z+$ $\left.i d p^{*} / d z\right)$, where $p$ is a capacity function, and $z$ is a local variable, we are able to establish a bound that implies that of Sario and can be smaller than $2+\eta$. It is also shown that the classical theorem of Picard holds unchanged for the meromorphic function $w_{p}$ provided that $w$ has maximum defect. In the concluding section a version of Milloux's extension of Nevanlinna's second main theorem is given for $W_{p}$ surfaces.
\end{abstract}

Let $W$ be an open Riemann surface and $w$ a meromorphic function on $W$. We shall restrict our attention to surfaces $W_{p}$ on which there exists a function $p$ with the following properties:

(1) $p$ is harmonic on $W-\left\{\zeta_{0}\right\}$, where $\zeta_{0} \in W$ is fixed,

(2) in a fixed parametric neighborhood $D$ containing $\zeta_{0}$, the function $u(\zeta)=p(\zeta)-\log \left|j(\zeta)-j\left(\zeta_{0}\right)\right|$ is harmonic and $u\left(\zeta_{0}\right)=0$; $j: D \rightarrow\{z:|z|<1\}$ is some fixed conformal homeomorphism,

(3) for $k \in\left[-\infty, k_{\beta}\right)-E$, where $E \subset\left[-\infty, k_{\beta}\right)\left(k_{\beta} \leqq \infty\right)$ is at most countable, $\Omega_{k}=p^{-1}([-\infty, k))$ is relatively compact and regularly embedded, and

(4) as $k$ tends to $k_{\beta}, \Omega_{k}$ exhausts $W$.

For example, it is known that all parabolic surfaces $\left(W \in O_{G}\right)$ are $W_{p}$ surfaces (see Z. Kuramochi [1], M. Nakai [3], L. Sario [6], [7]). For $W \in O_{G}, k_{\beta}=\infty$. A number $k^{\prime}$ is an element of $E$ if and only if $\partial p / \partial x=\partial p / \partial y=0$ for some point of the level line $\beta_{k^{\prime}}=p^{-1}\left(k^{\prime}\right)$. The number of such points in any $Q_{k}$ is clearly finite.

2A. For some $k, \Omega_{k}$ is regularly embedded and conformally equivalent to the unit disk. To simplify notation we shall assume that this

Received May 6, 1964. The results in this paper are part of the contents of the author's doctoral dissertation written under the direction of Prof. Leo Sario and sponsored in part by the U. S. Army Research Office (Durham), Grant DA-ARO(D)31-124-G40, University of California, Los Angeles. This manuscript was completed while the author was a research scholar of the Alexander von Humboldt Stiftung. at the Rhenish Friedrich Wilhelm University, Bonn, Germany. 
is the case for $k=0$.

L. Sario introduced the following proximity function

$$
\begin{aligned}
m(k, a) & =\frac{1}{2 \pi} \int_{\beta_{k}-\beta_{0}} \log \frac{1}{|w-a|} d p^{*}, \quad a \neq \infty, \\
m(k, \infty) & =m(k, w)=\frac{1}{2 \pi} \int_{\beta_{k}-\beta_{0}}^{+}+\frac{+}{+}|w| d p^{*}
\end{aligned}
$$

and the counting function

$$
N(k, a)=\int_{0}^{k} n(h, a) d h+n_{0}(a) k,
$$

where $n(k, a)$ counts (with multiplicities) the number of times $w$ assumes the value $a$ in $\Omega_{k}-\Omega_{0}$. The term $n_{0}(a)$ does the same with respect to $\Omega_{0}$. For $a=\infty$ one often writes $N(k, w)$. The characteristic function is $T(k, w)=m(k, w)+N(k, w)$, and the first main theorem is as follows:

THEOREM. For $w$ nonconstant and meromorphic on $W$ and for any value a

$$
m(k, a)+N(k, a)=T(k, w)+\varphi(k, a),
$$

where $\varphi(k, a)=O(1)$. Furthermore $\varphi(k, 0)=0$.

2B. The defect of a value $a$ is defined to be

$$
\delta(\alpha)=\lim _{k \rightarrow k_{\beta}} \frac{m(k, \alpha)}{T(k, w)}=1-\varlimsup_{k \rightarrow k_{\beta}} \frac{N(k, \alpha)}{T(k, w)} .
$$

Obviously $0 \leqq \delta(\alpha) \leqq 1$. If $e(k)$ is the Euler characteristic of $\Omega_{k}-\Omega_{0}$ and $E(k)=\int_{0}^{k} e(h) d h$, let

$$
\eta=\lim _{k \rightarrow k_{\beta}} \frac{E(k)}{T(k, w)} .
$$

L. Sario $[6,7]$ has shown that provided $T(k, w)$ grows fast enough

$$
\sum_{i=1}^{q} \delta\left(a_{i}\right) \leqq 2+\eta
$$

for any finite $q$, and that if $\eta<\infty$ the same inequality holds when the sum is taken over all $a$ for which $\delta(a)>0$.

3A. A function $w$ defined on a Riemann surface $W$ is meromorphic if for any parametric neighborhood $D, w \circ j^{-1}$ is meromorphic in $j(D)$. A derivative $w_{z}$ can be defined in $D$, but in general it cannot be extended to yield what one could call the derivative of $w ; w_{z}$ is covariant, 
not invariant. But the same is true of

$$
P_{z}=\frac{d p}{d z}+i \frac{d p^{*}}{d z}
$$

so the quotient

$$
w_{P}=\frac{w_{z}}{P_{z}}
$$

is invariant on $W$. We shall call this meromorphic function the $P$ derivative of $w$. The $P$-derivative plays a role similar to the one played by the ordinary derivative in the classical value distribution theory (cf. especially E. Ullrich [8], [9]).

There is a close relation between the growth of $w$ and of its $P$ derivative. Let $q>2$ finite complex numbers $a_{1}, \cdots, a_{q}, a_{i} \neq a_{j}, i \neq j$, be given. Form the function

$$
f(\zeta)=\sum_{j=1}^{q} \frac{1}{w(\zeta)-a_{j}},
$$

which for fixed $i$ may be written

$$
f(\zeta)=\frac{1}{w(\zeta)-a_{i}}\left[1+\sum_{\jmath \neq i} \frac{w(\zeta)-a_{i}}{w(\zeta)-a_{j}}\right] .
$$

Then set $\gamma=\min _{i \neq j}\left(\left|a_{i}-a_{j}\right|, 1\right)$, and suppose that $\zeta^{\prime} \in W$ is such that

$$
\left|w\left(\zeta^{\prime}\right)-a_{i}\right|<\frac{\gamma}{2 q}
$$

One easily establishes (see R. Nevanlinna [4], p. 242) that

$$
\log ^{+} \frac{1}{\left|w\left(\zeta^{\prime}\right)-a_{i}\right|}<\log ^{+}\left|f\left(\zeta^{\prime}\right)\right|+\log 3 .
$$

For $k>0$ let $\beta_{k i}^{\prime}$ be that portion of $\beta_{k}$ whose points satisfy (2), and let $\beta_{k i}^{\prime \prime}$ be the complement of $\beta_{k i}^{\prime}$ with respect to $\beta_{k}$. Clearly we have

$$
\begin{aligned}
m\left(k, a_{i}\right)= & \frac{1}{2 \pi} \int_{\beta_{k i}^{\prime}} \log \frac{1}{\left|w-a_{i}\right|} d p^{*} \\
& +\frac{1}{2 \pi}\left[\int_{\beta_{k i}^{\prime}}^{+} \stackrel{+}{\log } \frac{1}{\left|w-a_{i}\right|} d p^{*}-\int_{\beta_{0}} \stackrel{+}{\log } \frac{1}{\left|w-a_{i}\right|} d p^{*}\right],
\end{aligned}
$$

and after setting

$$
M=\frac{1}{2 \pi} \int_{\beta_{0}}+\stackrel{+}{\log }|f| d p^{*}-\frac{1}{2 \pi} \sum_{i=1}^{q} \int_{\beta_{0}}^{+} \log ^{+} \frac{1}{\left|w-a_{i}\right|} d p^{*}
$$

we conclude that 
$\sum_{i=1}^{q} m\left(k, a_{i}\right) \leqq \frac{1}{2 \pi} \int_{\beta_{k}-\beta_{0}} \stackrel{+}{\log }|f| d p^{*}+\log 3+q \log \frac{2 q}{\gamma}+M=m(k, f)+M^{\prime}$, $M^{\prime}$ constant.

Since $f=w_{P}^{-1} w_{P} f$, the inequality

$$
m(k, f) \leqq m\left(k, w_{P}^{-1}\right)+m\left(k, \sum \frac{w_{P}}{w-\alpha_{i}}\right) .
$$

is an obvious consequence of the subadditivity of $\log ^{+}$. By virtue of the first main theorem we can write

$$
m\left(k, w_{P}^{-1}\right)=T\left(k, w_{P}\right)-N\left(k, w_{P}^{-1}\right),
$$

and consequently

$$
T\left(k, w_{P}\right) \geqq m(k, f)+N\left(k, w_{P}^{-1}\right)-m\left(k, \sum \frac{w_{P}}{w-a_{i}}\right) .
$$

As in the proof of (3) one shows that

$$
m\left(k, w_{P}\right) \leqq m(k, w)+m\left(k, \frac{w_{P}}{w}\right),
$$

while the inequality

$$
N\left(k, w_{P}\right) \leqq N\left(k, w_{z}\right)+N\left(k, P_{z}^{-1}\right)
$$

follows from the definition.

Although $w_{z}$ is only locally defined, the multiplicity of a given pole of $w_{z}$ does not depend on any particular choice of the local variable. In fact, if $w$ has a pole of multiplicity $\nu$ at $\zeta^{\prime \prime}$, then $w_{z}$ has one of multiplicity $\nu+1$ at $\zeta^{\prime \prime}$. The well-defined function $\bar{n}(k, w)=n\left(k, w_{z}\right)-$ $n(k, w)$ therefore counts the poles of $w$ in $\Omega_{k}-\Omega_{0}$ exactly once. Set $\bar{N}(k, w)=N\left(k, w_{z}\right)-N(k, w)$.

A similar remark is valid with respect to the zeros of the covariant quantity $P_{z}$. The functions $n\left(k, P_{z}^{-1}\right)$ and $N\left(k, P_{z}^{-1}\right)$ are consequently well-defined. In $1 \mathrm{~B}$ we remarked that the number of points in $\Omega_{k}$ where $\partial p / \partial x=\partial p / \partial y=0$ is finite. In fact, this number does not exceed the Euler characteristic $e(k)$ of $\Omega_{k}-\Omega_{0}$. More exactly, if one applies the Riemann-Roch theorem to the double of $\bar{\Omega}_{k}$ and the meromorphic differential obtained by extending $P_{z} d z$ using the reflection principle, it can be shown that $e(k)=n\left(k, P_{z}^{-1}\right)$, and hence

$$
E(k)=N\left(k, P_{z}^{-1}\right)
$$

(cf. B. Rodin [5]). Inequalities (4) and (5) therefore imply the relation

$$
T\left(k, w_{P}\right) \leqq T(k, w)+\bar{N}(k, w)+E(k)+m\left(k, \frac{w_{P}}{w}\right) .
$$


We have established that for $q>2$ finite values $a_{1}, \cdots, a_{q}$

$$
\begin{aligned}
\sum_{i=1}^{q} m\left(k, a_{i}\right) & +N\left(k, w_{P}^{-1}\right)+R_{1}(k) \leqq T\left(k, w_{P}\right) \\
& \leqq T(k, w)+\bar{N}(k, w)+E(k)+R_{2}(k),
\end{aligned}
$$

where

$$
\begin{aligned}
& R_{1}(k)=-m\left(k, \sum \frac{w_{P}}{w-a_{i}}\right)-M^{\prime}, \\
& R_{2}(k)=m\left(k, \frac{w_{P}}{w}\right) .
\end{aligned}
$$

L. Sario [7] has shown that for any finite number $s$ of complex numbers $b_{j}$,

$$
m\left(k, \sum_{j=1}^{s} \frac{w_{P}}{w-b_{j}}\right)= \begin{cases}O(k+\log T(k, w)), & W \in O_{G} \\ O\left(\frac{1}{k_{\beta}-k_{i}}+\log T(k, w)\right), & W \notin O_{G}\end{cases}
$$

except for $k$ in a set of intervals $A_{\lambda}$ so small in total length that for $\lambda \geqq 0$ and $W \in O_{G}, \int_{\Delta_{\lambda}} e^{\lambda h} d h$ is finite, and if $W \notin O_{G}$ and $\lambda>0, \int_{\Delta_{\lambda}} e^{\lambda /\left(k_{\boldsymbol{\beta}}-h\right)} d h$ is finite.

We have proved the following

TheoRem. Let $q \geqq 3$ finite complex numbers $a_{1}, \cdots, a_{q}, a_{i} \neq a_{j}$, $i \neq j$, be given. For a function $w$ meromorphic on a Riemann surface $W$ the following inequalities are valid:

$$
\begin{aligned}
\sum_{i=1}^{q} m\left(k, a_{i}\right) & +N\left(k, w_{P}^{-1}\right)+R_{1}(k) \leqq T\left(k, w_{P}\right) \\
& \leqq T(k, w)+\bar{N}(k, w)+E(k)+R_{2}(k),
\end{aligned}
$$

where for $i=1,2$

$$
R_{i}(k)= \begin{cases}O(k+\log T(k, w)), & W \in O_{\theta} \\ O\left(\frac{1}{k_{\beta}-k}+\log T(k, w)\right), & W \notin O_{\theta}\end{cases}
$$

except for $k \in \Delta_{\lambda}$ such that if $W \in O_{G}$ and $\lambda \geqq 0, \int_{\Delta_{\lambda}} e^{\lambda h} d h<\infty$, and if $W \in O_{G}$, then $\int_{\lrcorner_{\lambda}} e^{\lambda /\left(k_{\beta}-h\right)} d h<\infty$ for $>0$.

As a corollary we have the following useful result:

CoROLlaRY. Let $q \geqq 3$ different finite complex numbers be given. Then for $w$ meromorphic on $W$ 


$$
\sum_{i=1}^{q} m\left(k, a_{i}\right) \leqq m\left(k, w_{P}^{-1}\right)-R_{1}(k)
$$

4A. We now turn to some consequences of the above results. In discussing defect values it is customary to impose the restriction

$$
\begin{array}{ll}
\frac{T(k, w)}{k} \rightarrow \infty \text { as } k \rightarrow \infty, & W \in O_{G}, \\
\left(k_{\beta}-k\right) T(k, w) \rightarrow \infty \text { as } k \rightarrow k_{\beta}, & W \notin O_{G},
\end{array}
$$

and we shall do so. This guarantees that $w$ behaves in some sense as it had an essential singularity on $\beta$ (cf. L. Sario [7]).

Inequality (7) implies that

$$
\begin{aligned}
m(k, w) & +\sum_{i=1}^{q} m\left(k, a_{i}\right) \leqq T(k, w)+m(k, w)+\bar{N}(k, w) \\
& +E(k)+R_{2}(k)-R_{1}(k),
\end{aligned}
$$

or that for any $q>3$ different values, finite or infinite,

$$
\sum_{i=1}^{q} m\left(k, a_{i}\right) \leqq 2 T(k, w)+E(k)+R_{2}(k)-R_{1}(k) .
$$

When one divides both sides of this relation by $T(k, w)$ and takes the lower limit as $k \rightarrow k_{\beta} \leqq \infty$, one concludes in view of (9) that

$$
\sum_{i=1}^{q} \delta\left(a_{i}\right) \leqq 2+\eta
$$

If $\eta<\infty$ it follows that there can be at most a countable number of $a^{\prime}$ s for which $\delta(a)>0$, and since (10) holds for all $q>3$ we have the result mentioned in $2 \mathrm{~B}$.

4B. However this result does not make full use of (7). Relation (8) implies that for $q>2$ different finite $a_{i}$

$$
\sum_{i=1}^{q} \frac{m\left(k, a_{i}\right)}{T(k, w)} \leqq \frac{m\left(k, w_{P}^{-1}\right)}{T\left(k, w_{P}\right)} \frac{T\left(k, w_{P}\right)}{T(k, w)}+o(1), \quad k \in \Delta_{\lambda}
$$

and (7) allows us to write

$$
\frac{T\left(k, w_{P}\right)}{T(k, w)} \leqq 1+\frac{\bar{N}(k, w)}{T(k, w)}+\frac{E(k)}{T(k, w)}+o(1), \quad k \in \Delta_{\lambda} .
$$

As a consequence of these inequalities we have

$$
\sum_{i=1}^{q} \lim _{\overline{k \rightarrow k_{\beta}}} \frac{m\left(k, a_{i}\right)}{T(k, w)} \leqq \varlimsup_{k \rightarrow k_{\beta}} \frac{m\left(k, w_{P}^{-1}\right)}{T\left(k, w_{P}\right)} \lim _{k \rightarrow h_{\beta}}\left(1+\frac{\bar{N}(k, w)}{T(k, w)}+\frac{E(k)}{T(k, w)}\right),
$$

and 


$$
\sum_{i=1}^{q} \delta\left(a_{i}\right) \leqq \Delta\left(O ; w_{P}\right)(2+\eta-\Theta(\infty)),
$$

where

$$
\begin{aligned}
\Delta\left(O ; w_{P}\right) & =\varlimsup_{k \rightarrow k_{\beta}} \frac{m\left(k, w_{P}^{-1}\right)}{T\left(k, w_{P}\right)}, \\
\Theta(\infty) & =1-\varlimsup_{k \rightarrow k_{\beta}} \frac{\bar{N}(k, w)}{T(k, w)} .
\end{aligned}
$$

Clearly the value of $\Delta\left(O ; w_{P}\right)$ lies between zero and one. Provided $\eta<\infty$ the defect sum on the left can be extended over all finite $a$, since the bound on the right of (14) is independent of $q$ :

$$
\sum_{a \neq \infty} \delta(a) \leqq \Delta\left(O ; w_{P}\right)(2+\eta-\Theta(\infty))
$$

The absence of $\delta(\infty)$ from the sum on the left does not decrease the usefulness of this result. Indeed, the contrary is true, since $\delta(\infty) \leqq \Theta(\infty) \leqq 1$.

In passing from (13) to (14) one has a choice: one can apply the upper limit to the first term on the right of (13) and the lower limit to the second or vice versa. If one makes the latter choice, the result is

$$
\sum_{a \neq \infty} \delta(\alpha) \leqq \delta\left(O ; w_{P}\right)(2+\bar{\eta}-\Theta(\infty))
$$

where

$$
\begin{aligned}
\bar{\eta} & =\varlimsup_{k \rightarrow k_{\beta}} \frac{E(k)}{T(k, w)}, \\
\partial\left(O ; w_{P}\right) & =\lim _{\overline{k \rightarrow k_{\beta}}} \frac{m\left(k, w_{P}^{-1}\right)}{T(k, w)} .
\end{aligned}
$$

in summary, we have this

Theorem. Provided $\eta$ is finite, the sum $\sum_{a \neq \infty} \hat{o}(a)$ for functions satisfying the growth condition (9) is bounded above by $\Delta\left(O ; w_{P}\right)(2+\eta-\Theta(\infty))\left(\right.$ or by $\left.\delta\left(O ; w_{P}\right)(2+\bar{\eta}-\Theta(\infty))\right)$.

It follows that if $\delta\left(O ; w_{P}\right)$ is zero and $\bar{\eta}$ finite, then any meromorphic function satisfying (9) can have at most one defect value, viz. $a=\infty$.

4C. That it is possible for a function meromorphic on an abstract Riemann surface to have more than the classical two Picard values, or that its defect sum can exceed two, is (except when the function grows too slowly) due to the generally more complicated topological 
structure of an abstract Riemann surface. The presence of $\eta$ in (10) makes this obvious. One might almost conjecture that the defect sum must be substantially over two for functions defined on more complicated surfaces. We shall show that this is not necessarily the case.

THEOREM. Let the nonconstant meromorphic function $w$ satisfy (9). If $\sum \delta(a)=2+\eta(\eta<\infty)$, then the defect sum for $w_{P}$ cannot reach three. In particular, $w_{P}$ can have no more than two Picard values.

If we let $\eta_{P}=\lim _{k \rightarrow k_{\beta}} E(k) / T\left(k, w_{P}\right)$, then the defect sum of $w_{P}$ is bounded above by $2+\eta_{P}$. We shall show that under the hypothesis of the theorem $\eta_{P}<1$.

From (8) it is clear that

$$
\sum_{a \neq \infty} \delta(a) \leqq \lim _{k \rightarrow k_{\beta}} \frac{T\left(k, w_{P}\right)}{T(k, w)},
$$

and using our hypothesis we conclude that

$$
\lim _{k \rightarrow k_{\beta}} \frac{T\left(k, w_{P}\right)}{T(k, w)} \geqq 1+\eta \text {. }
$$

By definition and the above estimate

$$
\begin{aligned}
\eta_{P}= & \lim _{k \rightarrow k_{\beta}} \frac{E(k)}{T\left(k, w_{P}\right)}=\lim _{k \rightarrow k_{\beta}}\left\{\frac{E(k)}{T(k, w)}\left[\frac{T\left(k, w_{P}\right)}{T(k, w)}\right]^{-1}\right\} \\
& \leqq \lim _{k \rightarrow k_{\beta}} \frac{E(k)}{T(k, w)}\left[\lim _{\frac{k \rightarrow k_{\beta}}{\beta}} \frac{T\left(k, w_{P}\right)}{T(k, w)}\right]^{-1} \leqq \frac{\eta}{1+\eta}<1,
\end{aligned}
$$

which establishes the theorem.

5A. The $n$th $P$-derivative is defined inductively as

$$
w_{P^{(n)}}=\frac{w_{P^{(n-1)_{z}}}}{P_{z}} .
$$

LEMMA.

$$
\bar{N}\left(k, w_{P^{(n)}}\right) \leqq \bar{N}(k, w)+n E(k) .
$$

The proof of this lemma is by induction and rests on the fact that $w$ has a pole at a point $\zeta \in W$ if and only if $w_{z}$ does.

THEOREM. Provided the meromorphic function $w$ and the surface $W$ are such that (9) holds and $\bar{\eta}<\infty$,

$$
T\left(k, w_{P(n)}\right) \leqq T(k, w)+n \bar{N}(k, w)+\frac{n}{2}(n+1) E(k)+R(k),
$$


where $R(k)=o(T(k, w))$ except for $k \notin \Delta_{\lambda}$ as in Theorem $3 A$.

For $n=1$, (18) is the second half of (7). Assume that (18) is valid for $n=m-1(m \geqq 2)$ :

(19) $T\left(k, w_{p^{(m-1)}}\right) \leqq T(k, w)+(m-1) \bar{N}(k, w)+\frac{m}{2}(m-1) E(k)+R(k)$,

where $R(k)=o(T(k, w))$ except in $\Delta_{\lambda}$. For the meromorphic function $w_{P(m-1)}$ inequality $(7)$ yields the relation

$$
T\left(k, w_{P^{(m)}}\right) \leqq T\left(k, w_{P^{(m-1)}}\right)+\bar{N}\left(k, w_{P^{(m-1)}}\right)+E(k)+R_{2}(k),
$$

where

$$
R_{2}(k)= \begin{cases}O\left(k+\log T\left(k, w_{P^{(m-1)}}\right)\right), & W \in O_{G} \\ O\left(\frac{1}{k_{\beta}-k}+\log T\left(k, w_{\boldsymbol{P}^{(m-1)}}\right)\right), & W \notin O_{G}\end{cases}
$$

except $\Delta_{\lambda}$.

Assume that $W \in O_{G}$. Since $\bar{\eta}<\infty$ there exists a $k_{0}$ with the property that $k>k_{0}$ implies $E(k) \leqq(\bar{\eta}+1) T(k, w)$, so for $k>k_{0}$ outside the exceptional set

$$
\begin{aligned}
T\left(k, w_{P^{(m-1)}}\right) & \leqq\left[m+\frac{m}{2}(m-1)(\bar{\eta}+1)\right] T(k, w)+o(T(k, w)) \\
& =O(T(k, w)) .
\end{aligned}
$$

Hence for $k$ outside an exceptional set as in Theorem $3 \mathrm{~A}$ and $W \in O_{G}$, $R_{2}(k)=o(T(k, w))$. A similar proof for $W \in O_{G}$ can be given. In view of Lemma 5A, (19) and (20) imply (18).

5B. Corollary $3 \mathrm{~A}$ can also be extended inductively.

THEOREM. Suppose that the meromorphic function $w$ and the surface $W$ are such that (9) holds and $\bar{\eta}<\infty$. For $q$ finite different $a_{i}$ and $k$ not in an exceptional set as in Theorem $3 A$

$$
\sum_{i=1}^{q} m\left(k, a_{i}\right) \leqq m\left(k, w_{P^{(n)}}^{-1}\right)+o(T(k, w)) .
$$

It follows from Theorem 5A that

$$
\varlimsup_{k \rightarrow k_{\beta}} \frac{T\left(k, w_{P(n)}\right)}{T(k, w)} \leqq(n+1)+\frac{n}{2}(n+1) \bar{\eta}-n \Theta(\infty) .
$$

By dividing both sides of (21) by $T(k, w)$ and then using (22) as in the proof of Theorem $4 \mathrm{~B}$, one shows that 


$$
\sum_{a \neq \infty} \delta(a) \leqq \delta\left(O ; w_{P(n)}\right)\left[(n+1)+\frac{n}{2}(n+1) \bar{\eta}-n \Theta(\infty)\right] .
$$

This establishes the following

THEOREM. Provided $w$ satisfies the growth condition (9) and $\bar{\eta}<\infty$, the vanishing of $\delta\left(O ; w_{P^{(n)}}\right)$ for any $n$ implies that $w$ has at most one defect value, viz. $a=\infty$. On the other hand, if $w$ has any finite defect value, then for every $n \geqq 1, \delta\left(O ; w_{P^{(n)}}\right)>0$, unless $\bar{\eta}=\infty$.

6A. H. Milloux [2] has studied the relations between the value distribution of a function $w$ meromorphic in the plane and that of its derivative $w_{z}$. In analogy we prove this

Theorem. Let $s>2, t>2$ finite complex numbers $a_{1}, \cdots, a_{s}$, $b_{1}, \cdots, b_{t}\left(b_{n} \neq 0\right)$ be given, $a_{i} \neq a_{j}$ and $b_{i} \neq b_{j}$ for $i \neq j$, and suppose $w$ is meromorphic on the Riemann surface $W$. Then

$$
\begin{aligned}
s t T(k, w) & \leqq \sum_{i=1}^{t} N\left(k, \frac{1}{w_{P}-b_{i}}\right)+t \sum_{j=1}^{s} N\left(k, \frac{1}{w-a_{j}}\right) \\
& -(t-1) N\left(k, \frac{1}{w_{P}}\right)+\bar{N}\left(k, w_{P}\right)+E(k)+R(k),
\end{aligned}
$$

where except for $k$ in a set $\Delta_{\lambda}$ as in Theorem $3 A R(k)=o(T(k, w))$ provided $w$ satisfies (9) and $\bar{\eta}<\infty$.

It follows from Theorem $3 \mathrm{~A}$ that

$$
\begin{aligned}
\sum_{i=1}^{t} m\left(k, \frac{1}{w_{P}-b_{i}}\right) & +m\left(k, \frac{1}{w_{P}}\right) \leqq T\left(k, w_{P}\right)+\bar{N}\left(k, w_{P}\right) \\
& +E(k)+R(k),
\end{aligned}
$$

By the first main theorem $T\left(k, w_{P}\right)=m\left(k, 1 / w_{P}\right)+N\left(k, 1 / w_{P}\right)$, and on applying this to (23) we obtain

$$
\sum_{i=1}^{t} m\left(k, \frac{1}{w_{P}-b_{i}}\right) \leqq N\left(k, \frac{1}{w_{P}}\right)+\bar{N}\left(k, w_{P}\right)+E(k)+R(k) .
$$

Another application of the first main theorem leads to the inequality

$$
\begin{aligned}
t T\left(k, w_{P}\right) & \leqq \sum_{i=1}^{t} N\left(k, \frac{1}{w_{P}-b_{i}}\right)+N\left(k, \frac{1}{w_{P}}\right)+\bar{N}\left(k, w_{P}\right) \\
& +E(k)+R(k)+O(1) .
\end{aligned}
$$

The left half of the main inequality (7) together with the first main theorem imply the relation

$$
s T(k, w) \leqq T\left(k, w_{P}\right)+\sum_{j=1}^{s} N\left(k, \frac{1}{w-a_{j}}\right)-N\left(k, \frac{1}{w_{P}}\right)+R_{1}(k) .
$$


On multiplying (25) by $t$ and substituting from (24) we have the desired inequality. The argument of $5 \mathrm{~A}$ shows that the resultant remainder term is $o(T(k, w)), k \notin \Delta_{\lambda}$.

6B. The relative and absolute defects of H. Milloux [2] can also be defined on an open Riemann surface $W$. We define the relative defect of a value $a$ with respect to the function $w$ as

$$
\begin{aligned}
& \grave{o}_{r}(a)=1-\varlimsup_{k \rightarrow k_{\beta}} \frac{N\left(k, \frac{1}{w_{P}-a}\right)}{T(k, w)}, a \text { finite, } \\
& \hat{o}_{r}(\infty)=1-\varlimsup_{k \rightarrow k_{\beta}} \frac{N\left(k, w_{P}\right)}{T(k, w)} .
\end{aligned}
$$

The absolute defect is

$$
\begin{aligned}
& \delta_{a}(a)=1-\varlimsup_{k \rightarrow k_{\beta}} \frac{N\left(k, \frac{1}{w_{P}-a}\right)}{T\left(k, w_{P}\right)}, a \text { finite } \\
& \delta_{a}(\infty)=1-\varlimsup_{k \rightarrow k_{\beta}} \frac{N\left(k, w_{P}\right)}{T\left(k, w_{P}\right)} .
\end{aligned}
$$

As an easy consequence of (12) one establishes the following relation:

Theorem. Provided $w$ satisfies (9) and $\bar{\eta}<\infty$,

$$
-(\bar{\eta}+1)+\left[(2+\bar{\eta}) \delta_{n}(a)\right] \leqq \delta_{r}(a) \leqq 1 .
$$

\section{REFERENCES}

1. Z. Kuramochi, Evans's theorem on abstract Riemann surfaces with nullboundaries. I, II, Proc. Japan Acad. 32 (1956), 1-6, 7-9.

2. H. Milloux, Les dérivées des fonctions méromorphes et la théorie des défauts, Ann. Sci. École Norm. Sup. Ser. 3, 63 (1946), 289-316.

3. M. Nakai, Evans potential, Proc. Japan Acad. 38 (1962), 624-629.

4. R. Nevanlinna, Eindeutige analytische Funktionen, 2nd ed., Springer Verlag, Berlin-Göttingen-Heidelberg, (1953), 379 pp.

5. B. Rodin, Reproducing formulas on Riemann surfaces, Doctoral dissertation, University of California, Los Angeles, (1961), $71 \mathrm{pp}$.

6. L. Sario, Picard's great theorem on Riemann surfaces, Amer. Math. Monthly 69 (1962), 598-608.

7. - Meromorphic functions and conformal metrics on Riemann surfaces, Pacific J. Math. 12 (1962), 1079-1098.

8. E. Ullrich, Über die Ableitung einer meromorphen Funktion, Sitzgber. preuss. Akad. Wiss., Math.-Phys. Kl. (1929), 592-608.

9. - Über den Einfluss der Verzweigtheit einer Algebroide auf ihre Wertverteilung, J. f. Math. 167 (1932), 198-220. 


\section{PACIFIC JOURNAL OF MATHEMATICS}

\section{EDITORS}

\section{H. SAMELSON}

Stanford University

Stanford, California

R. M. Blumenthal

University of Washington

Seattle, Washington 98105

\author{
*J. DugundJI \\ University of Southern California \\ Los Angeles, California 90007 \\ RICHARD ARENS \\ University of California \\ Los Angeles, California 90024
}

\section{ASSOCIATE EDITORS}
E. F. BECKENBACH
B. H. NeUManN
F. WolF
K. YosIDA

\section{SUPPORTING INSTITUTIONS}

UNIVERSITY OF BRITISH COLUMBIA
CALIFORNIA INSTITUTE OF TECHNOLOGY
UNIVERSITY OF CALIFORNIA
MONTANA STATE UNIVERSITY
UNIVERSITY OF NEVADA
NEW MEXICO STATE UNIVERSITY
OREGON STATE UNIVERSITY
UNIVERSITY OF OREGON
OSAKA UNIVERSITY
UNIVERSITY OF SOUTHERN CALIFORNIA

UNIVERSITY OF BRITISH COLUMBIA

UNIVERSITY OF CALIFORNIA

MONTANA STATE UNIVERSITY

NEW MEXICO STATE UNIVERSITY

OREGON STATE UNIVERSITY

OSAKA UNIVERSITY

UNIVERSITY OF SOUTHERN CALIFORNIA

\author{
STANFORD UNIVERSITY \\ UNIVERSITY OF TOKYO \\ UNIVERSITY OF UTAH \\ WASHINGTON STATE UNIVERSITY \\ UNIVERSITY OF WASHINGTON \\ AMERICAN MATHEMATICAL SOCIETY \\ CHEVRON RESEARCH CORPORATION \\ TRW SYSTEMS \\ NAVAL ORDNANCE TEST STATION
}




\section{Pacific Journal of Mathematics \\ Vol. 17, No. $1 \quad$ January, 1966}

Carlos Jorge Do Rego Borges, On stratifiable spaces ................ 1

Felix Earl Browder, Topological methods for non-linear elliptic equations of

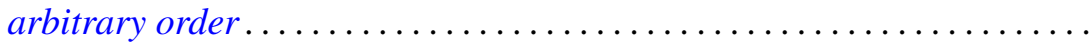

Gustave Choquet, Harry Corson and Victor Klee, Exposed points of convex

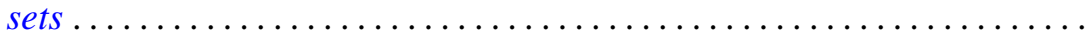

Phillip Emig, Remarks on the defect sum for a function meromorphic on an open Riemann surface ................................ 45

Ruth Goodman, A certain class of polynomials .................. 57

Sidney (Denny) L. Gulick, The bidual of a locally multiplicatively-convex

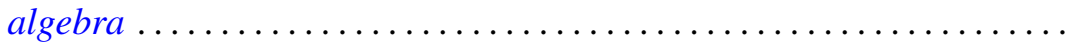

Eugene Carlyle Johnsen, Integral solutions to the incidence equation for finite projective plane cases of orders $n \equiv=2(\bmod 4) \ldots \ldots \ldots \ldots . .67$

Charles N. Kellogg, Centralizers and $H^{*}$-algebras .................. 121

Michael Lodato, On topologically induced generalized proximity relations. II .......................................... 131

P. H. Maserick, Half rings in linear spaces ..................... 137

Kathleen B O'Keefe, On a problem of J. F. Ritt .................... 149

Galen Lathrop Seever, Nonnegative projections on $C_{0}(X) \ldots \ldots \ldots \ldots$

Lawrence A. Shepp, Gaussian measures in function space ............ 167

Robert Charles Thompson, Classes of definite group matrices ........... 175 